\title{
Immune checkpoint inhibitors for treatment of small-cell lung cancer: a systematic review and meta-analysis
}

\author{
Zhicheng Niu ${ }^{1}$, Shenghu Guo ${ }^{1}$, Jing Cao ${ }^{1}$, Yuehua Zhang ${ }^{1}$, Xiaojin Guo ${ }^{1}$, Francesco Grossi ${ }^{2}$, \\ Yoshinobu Ichiki ${ }^{3,4}$, You Li ${ }^{1}$, Zhiyu Wang ${ }^{1}$ \\ ${ }^{1}$ The Fourth Hospital of Hebei Medical University, Shijiazhuang, China; ${ }^{2}$ Medical Oncology Unit, Fondazione IRCCS Ca' Granda Ospedale \\ Maggiore Policlinico, Milano, Italy; ${ }^{3}$ Department of General Thoracic Surgery, National Hospital Organization, Saitama Hospital, Wako, Japan; \\ ${ }^{4}$ Second Department of Surgery, University of Occupational and Environmental Health, School of Medicine, Kitakyushu, Japan \\ Contributions: (I) Conception and design: Z Wang, Z Niu; (II) Administrative support: Z Wang, S Guo; (III) Provision of study materials or patients: \\ Y Zhang, X Guo, Z Wang; (IV) Collection and assembly of data: Z Niu, J Cao, Y Li; (V) Data analysis and interpretation: Z Niu, F Grossi, Y Ichiki; (VI) \\ Manuscript writing: All authors; (VII) Final approval of manuscript: All authors. \\ Correspondence to: Zhiyu Wang. The Fourth Hospital of Hebei Medical University, Shijiazhuang, China. Email: 327369979@qq.com.
}

Background: Small cell lung cancer (SCLC) is a very aggressive and proliferative disease, with little progress being having made for its treatment in decades. Our goal was to evaluate the effect of immune checkpoint inhibitors (ICIs) and identify optimal first-line interventions for the treatment of SCLC.

Methods: A systematic literature search of the Cochrane Library, PubMed and oncology conference proceedings were conducted. Randomized trials evaluating ICIs for SCLC were included. We use the risk of bias tool in RevMan 5.3 to assess the quality of studies. We used Stata version 15.0 to carry out data direct comparison and $\mathrm{R}$ version 4.0.2 to conduct the Bayesian network analysis.

Results: A total of 16 relevant clinical trials comprising 4,476 patients were included. We found the magnitude of efficacy for ICIs as first-line therapy conferred a statistically significant benefit in overall survival (OS) and progression-free survival compared to chemotherapy alone. The results were $0.82(95 \%$ CI, 0.76-0.89, $\mathrm{P}<0.001)$ and 0.80 (95\% CI, 0.74-0.86, $\mathrm{P}<0.001)$. For objective response rate (ORR), the result $(1.13,95 \% \mathrm{CI}, 0.97-1.31, \mathrm{P}=0.109)$ was not significant. In the second-line and maintenance treatment, no additional benefit was observed. With regard to safety, results showed that for all grades of AEs and grades 3-4 AEs, the pooled results were 1.36 (95\% CI: 0.50-3.70; P=0.543) and 1.35 (95\% CI: 0.58-3.15; $\mathrm{P}=0.484$ ) respectively. In addition, the indirect comparison results showed that nivolumab combined with chemotherapy led to the most significant improvement in OS, while durvalumab combined with chemotherapy was a more efficacious therapy for improving ORR compared with the other interventions; the probability were the best treatments was $73.93 \%$ and $81 \%$ respectively.

Discussion: Our results showed ICIs combined with etoposide and platinum-based drugs as first-line treatment of SCLC have benefits for patients and there was no evidence of a significant difference in efficacy among the different ICI drugs used for the first-line therapy. As for toxicity, the ICIs did not increase the frequency AEs for patients. However, as some studies are ongoing and the full data have still not been reported, our conclusions may not be completely representative.

Keywords: Immune checkpoint inhibitors (ICIs); small cell lung cancer (SCLC); meta-analysis; Bayesian network analysis

Submitted Nov 18, 2020. Accepted for publication Apr 22, 2021.

doi: $10.21037 / \mathrm{atm}-21-1423$

View this article at: http://dx.doi.org/10.21037/atm-21-1423 


\section{Introduction}

Over the past two decades, the incidence and mortality of lung cancer have ranked first in the world, increasing each year, and seriously threatening people's health. Small cell lung cancer (SCLC) accounts for about $15 \%$ of all lung cancer cases. Although the incidence is low, it has the characteristics of short doubling time, high invasiveness, and early widespread metastasis (1). The staging of SCLC is a key factor in determining treatment and prognosis. According to the staging method of the Veterans Administration Lung Study Group (VALSG), SCLC can be divided into two categories: limited-stage (LS-SCLC) and extensive-stage (ES-SCLC) around two third of the patients. The characteristic of LS-SCLC is that the tumor is confined to one side of the chest cavity, involves supraclavicular or anterior scalene lymph node metastasis and ipsilateral pleural effusion, and can be included in a radiotherapy field. LS-SCLC is mainly treated with concurrent radiotherapy and chemotherapy, or chemotherapy and sequential radiotherapy treatment, and prophylactic cranial irradiation (PCI) should be administered in patients with response to treatment. However, these therapies have a limited therapeutic effect on patients, and the 5-year survival rate is about $20-25 \%$. Extensive stage refers to diseases that cannot be classified as limited stage, and include malignant pleural or pericardial effusion and blood metastasis (2). For ES-SCLC, standard platinum-containing chemotherapy is initially effective, but recurrence and chemotherapy resistance always occur, and the median survival time is often less than 1 year. PCI is indicated also in patients with ES-SCLC that responded to chemotherapy and thoracic radiotherapy is indicated in patients with a residual thoracic disease after chemotherapy. With regards to the timing of thoracic radiotherapy for ES-SCLC, Luo's (3) study showed no evidence of a difference in overall survival, progressionfree survival, and locoregional recurrence-free survival between patients who received early ( $\leq 3$ cycles of induction chemotherapy) or late ( $>3$ cycles) thoracic radiotherapy. However, this study is retrospective, and the results need to be confirmed by more prospective studies. For many years, the combination of etoposide and platinum-based drugs (EP) has been the standard first-line treatment for ES-SCLC, with an objective response rate (ORR) of about $40-70 \%$. The second-line treatment of topotecan or irinotecan is not satisfactory. Thus far, molecular targeted therapy has not proven to have any substantial effect on SCLC (4).

The occurrence of SCLC is closely related to tobacco consumption, with smokers accounting for $95 \%$ of SCLC cases. Smoking causes DNA damage, genome distortion, and genome instability in tumor cells. In relation to smoking exposure, a high neoantigen load can be established (5). In solid tumors of lung cancer, the number of antigen mutations is the trigger of tumor-specific $\mathrm{T}$ lymphocyte response. Activating T lymphocytes can kill tumor cells and activate the immune system to achieve protection (6). In addition, studies have found that the survival rate of patients with a higher effector T-cell to regulatory $\mathrm{T}$-cell ratio in the blood is significantly higher that in patients with a lower number (7). SCLC has the characteristics of high mutation burden, making immunotherapy another effective treatment method for SCLC (8).

At present, in solid tumors, successful immunotherapy mainly refers to inhibiting the combination of immune checkpoints and their ligands, reducing immune tolerance, improving immune cell activity, and attacking malignant cells (9). Cytotoxic T lymphocyte-associated antigen-4 (CTLA-4) is an immunosuppressive regulator and a transmembrane receptor on $\mathrm{T}$ cells. It induces T lymphocyte cell anergy by binding to $\mathrm{B} 7$ molecules. Another negative regulator is programmed cell death protein 1 (PD-1), which is expressed on the surface of activated $\mathrm{T}$ cells, $\mathrm{B}$ cells, and macrophages. The binding of PD-1 to its ligand, programmed cell death ligand-1 (PDL1), leads to local immunosuppression, and the protection of the tumor from immune surveillance. Therefore, immune checkpoint inhibitors (ICIs) can play an effective role in controlling and eradicating cancer by negating the above mechanisms (10). Currently, the ICIs drugs researched in clinical trials of SCLC mainly consist of PD-1 inhibitors, including pembrolizumab (Pem) and nivolumab (Niv); PDL1 inhibitors, including atezolizumab (Ate) and durvalumab (Dur); and CTLA-4 inhibitors, including ipilimumab (Ipi) and tremelimumab (Tre) (11-26). In some of these clinical trials, ICI used either as monotherapy or combined with chemotherapy are more effective for survival benefits when compared to standard chemotherapy, but the majority of patients are not responsive. However, determining the degree of efficacy for the application of ICIs in SCLC requires further evaluation and which strategy is the optimal choice as first-line therapy for SCLC needs to be identified.

Toward this end, we performed a systematic review and meta-analysis of relevant randomized clinical trials concerning the efficacy of ICIs in SCLC. These data were indirectly compared through the Bayesian statistical methods. We present the following article in accordance 
with the PRISMA reporting checklist (available at http:// dx.doi.org/10.21037/atm-21-1423).

\section{Methods}

\section{Protocol and registration}

This study was conducted according to the PRISMA checklist (Preferred Reporting Items for Systematic Reviews and Meta-Analyses) and was submitted to PROSPERO (the International Prospective Register of Systematic Review; no. CRD42020211223) $(27,28)$. There was no any amendments in the protocol.

\section{Search strategies and literatures selection}

This review systematically searched the Cochrane Library and Medline (through PubMed) for articles published until October 2020. Furthermore, abstract listings between 2018 to 2020 from major oncology conference proceedings, including the American Society of Clinical Oncology meetings, the European Society for Medical Oncology meetings, and the World Conference on Lung Cancer, were browsed. The search strategies are summarized in Table S1. The keywords for our search primarily consisted of the following: "immune checkpoint inhibitors," "small cell lung cancer," and "randomized controlled trials". The search strategies adopted a combination of MESH (Medical Subject Headings) subject words and free words, connected with Boolean operators, "AND" or "OR", accordingly. All search strategies were determined after multiple presearches, and the final search strategies employed were agreed upon unanimously by all researchers.

Materials that met the following criteria were included, and those that did not meet the requirements were excluded: (I) patients had pathologically confirmed limited or extensive SCLC; (II) the intervention included one or more ICI drugs; (III) the treatment of the control group was conventional chemotherapy; (IV) we could extracted data for hazard ratios (HRs) of overall survival (OS) and progression-free survival (PFS) with corresponding 95\% confidence intervals (CIs), ORR, and the incidence of adverse events (AEs); (V) the study type was a randomized controlled trial (RCT).

Literature selection was independently conducted by 2 investigators, and any disagreements were resolved through discussion.

\section{Quality assessment}

We use the risk of bias (RoB) tool in RevMan 5.3 (Cochrane) to access the quality of the literature (29). Seven key domains were evaluated: (I) random sequence generation: describes the method used to generate the allocation sequence in sufficient detail to allow an assessment of whether it should produce comparable groups; (II) allocation concealment: describes the method used to conceal the allocation sequence in sufficient detail to determine whether intervention allocations could have been foreseen in advance of, or during, enrolment; (III) blinding of participants and personnel: describes all measures used, if any, to blind study participants and personnel from knowledge of which intervention a participant received; (IV) blinding of outcome assessment: describe all measures used, if any, to blind outcome assessors from knowledge of which intervention a participant received; $(\mathrm{V})$ incomplete outcome data: describes the completeness of outcome data for each main outcome, including attrition and exclusions from the analysis; (VI) selective reporting: states how the possibility of selective outcome reporting was examined by the review authors, and what was found; (VII) other biases: states any important concerns about bias not addressed in the other domains in the tool. Each item was rated as "low risk of bias" (all key domains indicated as low risk), "high risk of bias" (one or more key domains indicated as high risk), or "unevaluable risk of bias". The process was independently conducted by 2 investigators, with any disagreements being solved by consensus.

\section{Data extraction}

The information extracted using a standardised data collection form from all the RCTs included the name of the first author, year of publication, title of the trial, treatments used in each arm, line of treatment, number of patients, median follow-up duration, HR for OS and PFS with corresponding $95 \%$ CIs, ORR, and the incidence of AEs. In order to avoid errors in the data extraction process, 2 independent researchers extracted the data and resolved their differences through discussion. When we needed information that were unclear, we contacted the corresponding authors to request help. The extracted data are displayed in Table 1.

\section{Statistical analysis}

Meta-analysis is a method that uses various statistical 
Table 1 Baseline characteristics and outcomes of included trials

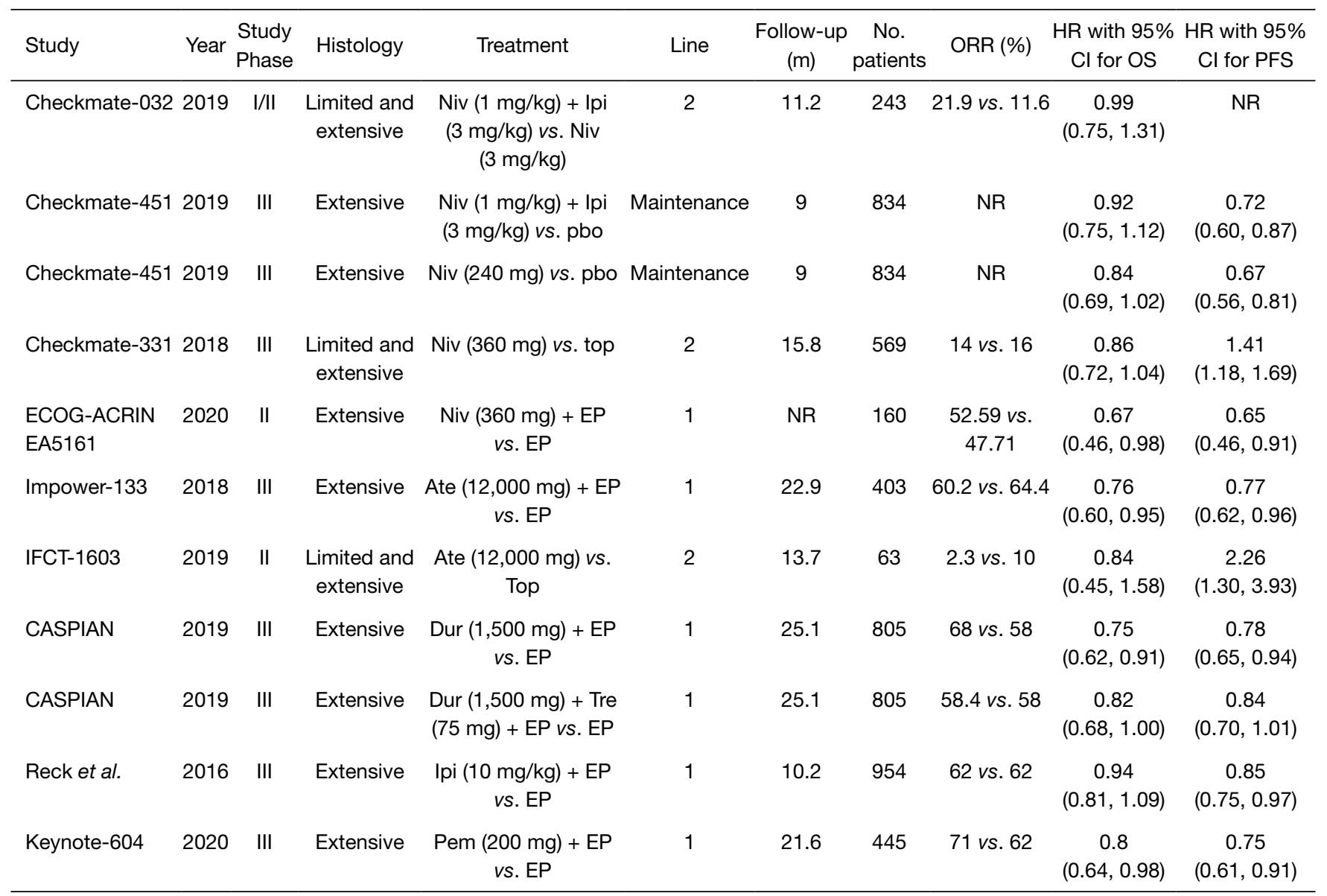

NR, not reported. EP, etoposide and platinum-based drugs. Pem + EP, pembrolizumab combined with EP. Ate + EP, atezolizumab combined with EP. Ipi + EP, ipilimumab combined with EP. Dur + Tre + EP, durvalumab and tremelimab combined with EP. Dur + EP, durvalumab combined with EP, and Niv + EP, nivolumab combined with EP. Top, topotecan. Ate, atezolizumab. Niv, nivolumab. Niv + Ipi, nivolumab combined with ipilimumab. pbo, Placebo. HR, hazard ratios. Cls, confidence intervals. OS, overall survival. PFS, progress-free survival. ORR, objective response rate.

methods to collect, merge and statistically analyze different research results. The potential advantages of meta-analysis include increased testing power, improved accuracy, answering questions that a single study cannot answer, and resolving disputes caused by conflicting views. We used Stata version 15.0 (StataCorp) to carry out data direct comparison. For binary variables (such as ORR), we use the odds ratio (OR) as the effect size. For time-survival variables (such as OS), we use HR and its corresponding 95\% CIs as the effect size. The process was independently conducted by 2 investigators, with any disagreements being solved by consensus. The statistical analysis results were display using a standardised table. In this meta-analysis, the quantity $I^{2}$ (range, $0-100 \%$ ) was used to measure the degree of heterogeneity between studies (30,31). Heterogeneity $\mathrm{I}^{2}$ $\geq 50 \%$ and $\mathrm{P}$ value $<0.05$ indicated significant heterogeneity in the study. If there was no statistical heterogeneity between the results of the studies, the fixed effects model (Mantel-Haenszel method) was used; if there was statistical heterogeneity between the results of each study, then further step-by-step analysis of the source of heterogeneity, such as subgroups analysis, was applied; after excluding the influence of obvious clinical heterogeneity, the random effects model (Dersimonian-Laird method) was used to conduct the meta-analysis. We further used a funnel plot to detect publication bias. If the funnel plot showed obvious asymmetry, we used Egger's test to assess the asymmetry of the funnel plot. If a small-sample research effect was 


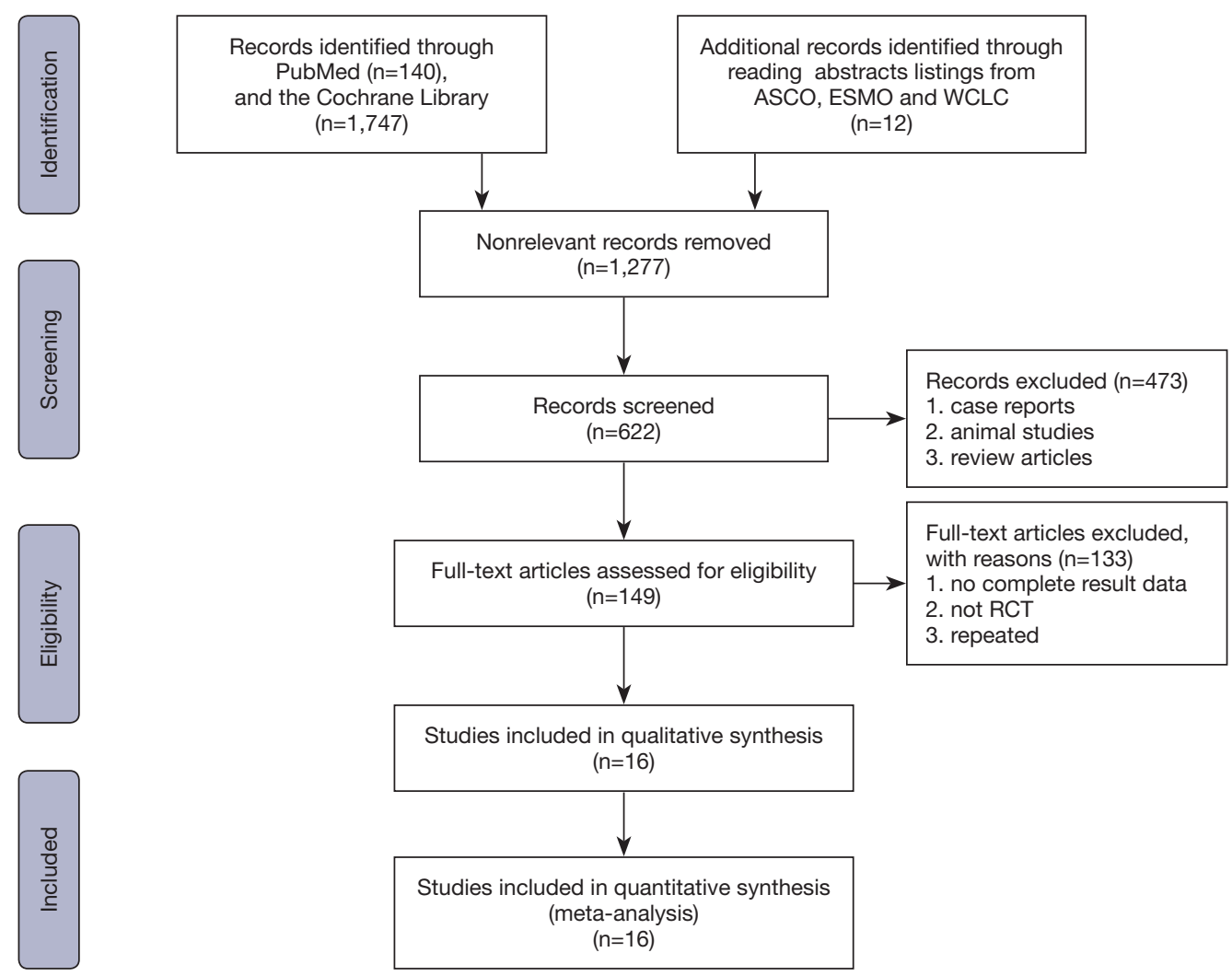

Figure 1 The flow diagram of the literature search and selection process. ASCO, American Society of Clinical Oncology meetings; ESMO, the European Society for Medical Oncology meetings; WCLC, the World Conference on Lung Cancer.

detected, we conducted a sensitivity analysis. The process was independently conducted by 2 investigators to ensure the accuracy of the results.

Network meta-analysis (NMA) is based on the traditional analysis of the results of indirect comparisons. When a certain type of disease has multiple treatment plans, NMA combined with the evidence of direct comparison and indirect comparison can be used to compare multiple interventions at the same time (32). Based on the Bayesian framework and the Markov Chain Monte Carlo (MCMC) method, we perform indirect comparisons of various treatment measures. For each specific parameter, we inferred the posterior probability according to the prior probability. In this study, the OR value and the HR were used as statistical indicators of effect, with the results being reported as median and $95 \%$ credible interval (CrI). Consequently, 4 Markov chains were used for the initial value setting, and the total number of iterations was set to 200,000 , with the first 40,000 being used for annealing to eliminate the influence of the initial value. Statistical validity was guaranteed when the $95 \%$ CrI did not include 1 (33). The Brooks-Gelman-Rubin diagnostic method was used to evaluate the degree of iterative convergence of the model, which was specifically reflected in the evaluation of the trajectory map and density map through intuitive vision (34). To summarize probabilities of each available treatment, the surfaces under the cumulative ranking curve (SUCRA) were calculated. The range of SUCRA is $0-100 \%$; when the SUCRA value is $100 \%$, this indicates that the intervention is the best of the intervention measures, when the value is $0 \%$, it is the worst (35). We used "rjags" and the "gemtc" packages in $\mathrm{R}$ version 4.0.2 ( $\mathrm{R}$ Project for Statistical Computing) to conduct the analyses (36).

\section{Results}

\section{Literature selection and basic characteristics}

The literature search process and results are summarized in Figure 1. The search in electronic databases and 


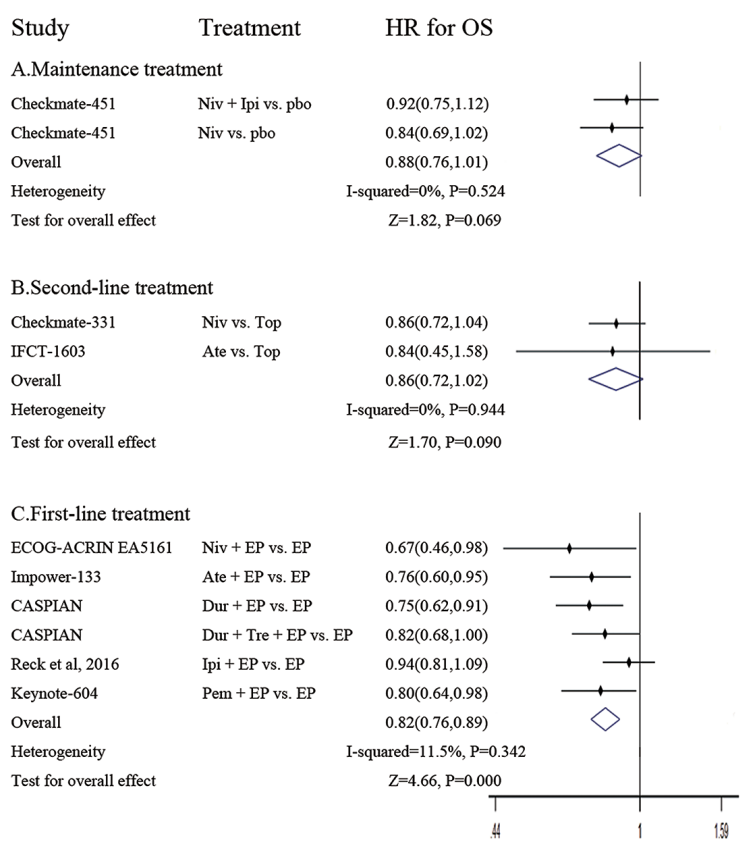

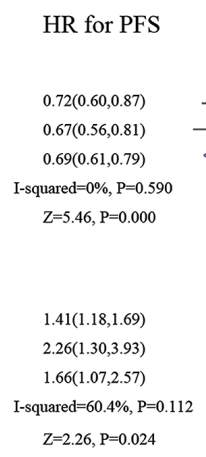
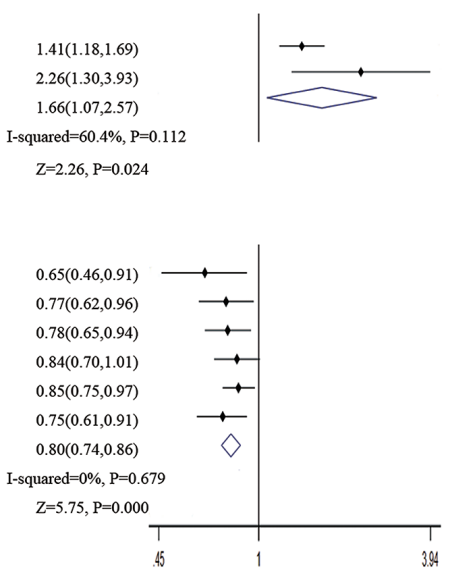

OR for ORR
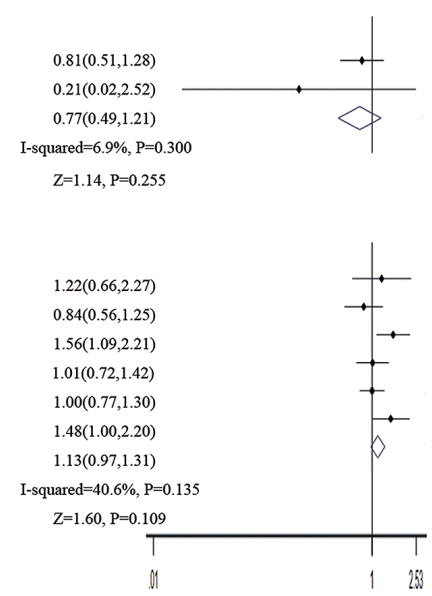

Figure 2 Direct comparison for OS, PFS, and ORR. OS, overall survival; PFS, progression-free survival; ORR, objective response rate.

meeting abstracts yielded 1,899 citations, of which 1,277 were not relevant. After reading the titles and abstracts of the remaining documents, those that did not meet the inclusion criteria (such as not RCT, no complete result data) were excluded. Finally, 16 studies, comprising 4,476 patients, were included. Regarding the lines of treatment, 9 treatments were first-line therapy, including EP alone or combined with Pem + EP $(17,18)$, Ate + EP $(15,16,20)$, ipilimumab (Ipi + EP) (23), Dur and tremelimab (Dur + Tre + EP), Dur + EP $(21,22,25)$, and Niv + EP (14). For the second-line treatment, 3 treatments in addition to standard topotecan chemotherapy were found: Ate (19), Niv (12), and nivolumab combined with ipilimumab (Niv + Ipi) $(10,11,24)$. For maintenance treatment, nivolumab combined with ipilimumab (Niv + Ipi) and Niv, were retrieved (13). The baseline characteristics and extracted data of these 16 studies are shown in Table 1.

\section{Direct comparison for ICI-based treatment and chemotherapy}

Results of direct comparison for OS, PFS, and ORR are shown in Figure 2. Overall, the addition of ICIs to standard chemotherapy showed significant advantage over chemotherapy alone as first-line therapy for OS and PFS.
The pooled HR were 0.82 (95\% CI, 0.76-0.89, $\mathrm{P}<0.001)$ and $0.80(95 \% \mathrm{CI}, 0.74-0.86, \mathrm{P}<0.001)$ respectively. With regard to ORR, the pooled OR was 1.13 (95\% CI, 0.971.31, $\mathrm{P}=0.109$ ), which was not significant. There was no obvious heterogeneity between the trials. In the secondline treatment, the effect of ICI monotherapy was not found to be more effective than topotecan for OS, PFS or ORR. The pooled HR of OS and PFS were 0.86 (95\% CI, $0.7-1.02, \mathrm{P}=0.090)$ and 1.66 (95\% CI, 1.07-2.57, $\mathrm{P}=0.024)$. For ORR, the pooled OR was 0.77 (95\% CI, 0.49-1.21, $\mathrm{P}=0.255)$. When ICIs were used in maintenance therapy, we also found that ICIs did not significantly improve OS, with the pooled HR of OS being 0.88 (95\% CI, 0.76-1.01, $\mathrm{P}=0.069)$. However, for PFS, the pooled HR was $0.69(95 \%$ CI, 0.61-0.79, $\mathrm{P}=0.001$ ), which was significant.

In order to explore the impact of population baseline characteristics on immunotherapy, we also performed subgroup analysis for OS according to patients' characteristics. Results were showed in Figure 3. For each of the subgroups (age, sex, liver metastasis, choice of platinum drugs and performance status), the magnitude of efficacy of ICIs was statistically significant benefit in OS than chemotherapy alone. But for patients with brain metastasis and those who do not smoke, the combined therapy was not likely to have benefits. 

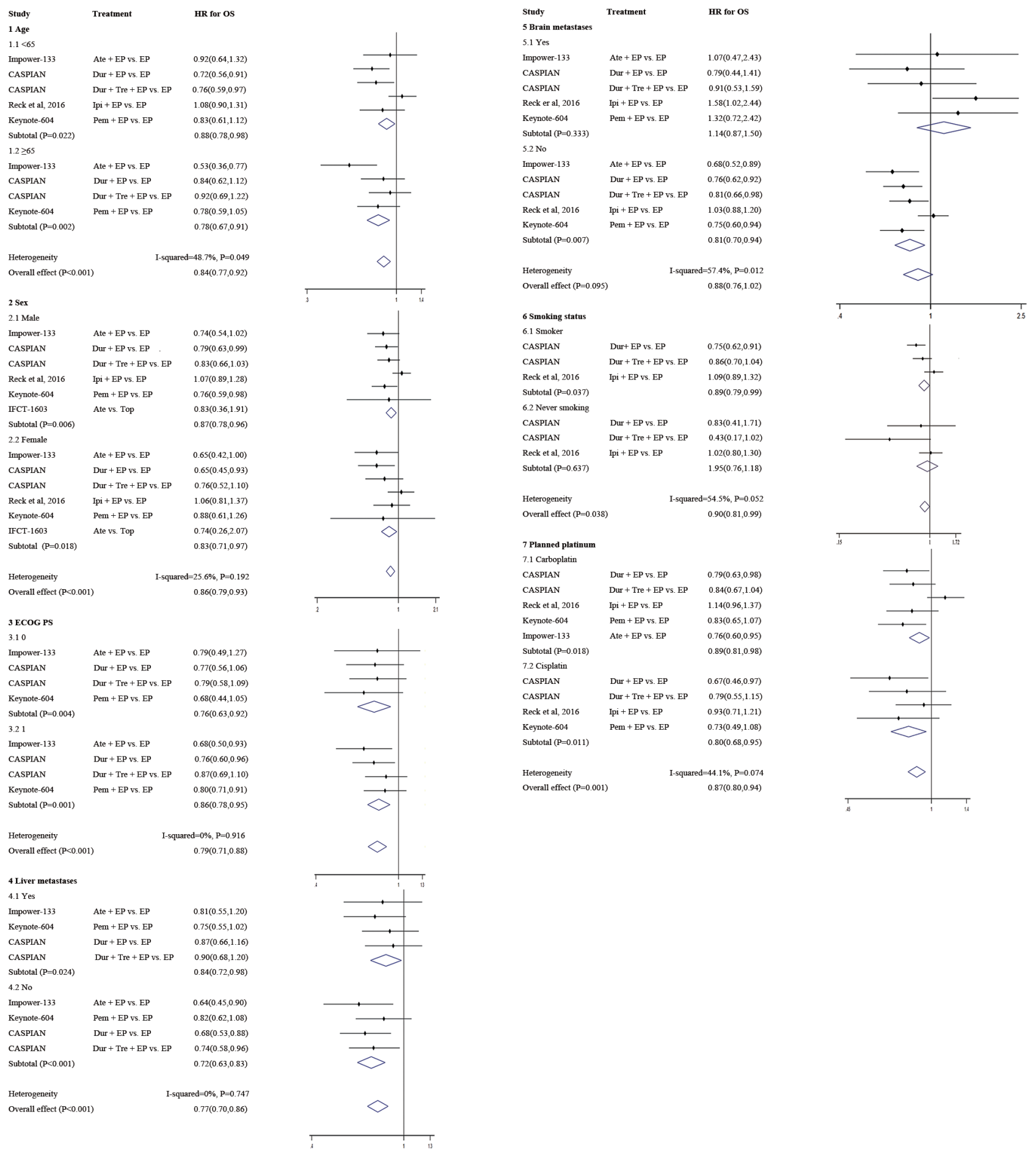

Figure 3 Subgroup analysis for overall survival according to patients' characteristics. EP, etoposide and platinum-based drugs; Pem + EP, pembrolizumab combined with EP; Ate + EP, atezolizumab combined with EP; Ipi + EP, ipilimumab combined with EP; Dur + Tre + EP, durvalumab and tremelimab combined with EP; Dur + EP, durvalumab combined with EP; Niv + EP, nivolumab combined with EP; Top, topotecan; Ate, atezolizumab; Niv, nivolumab; Niv + Ipi, nivolumab combined with ipilimumab; HR, hazard ratio; OS, overall survival; ECOG, Eastern Cooperative Oncology Group; PS, performance status. 
Table 2 Adverse events of control and experimental arms

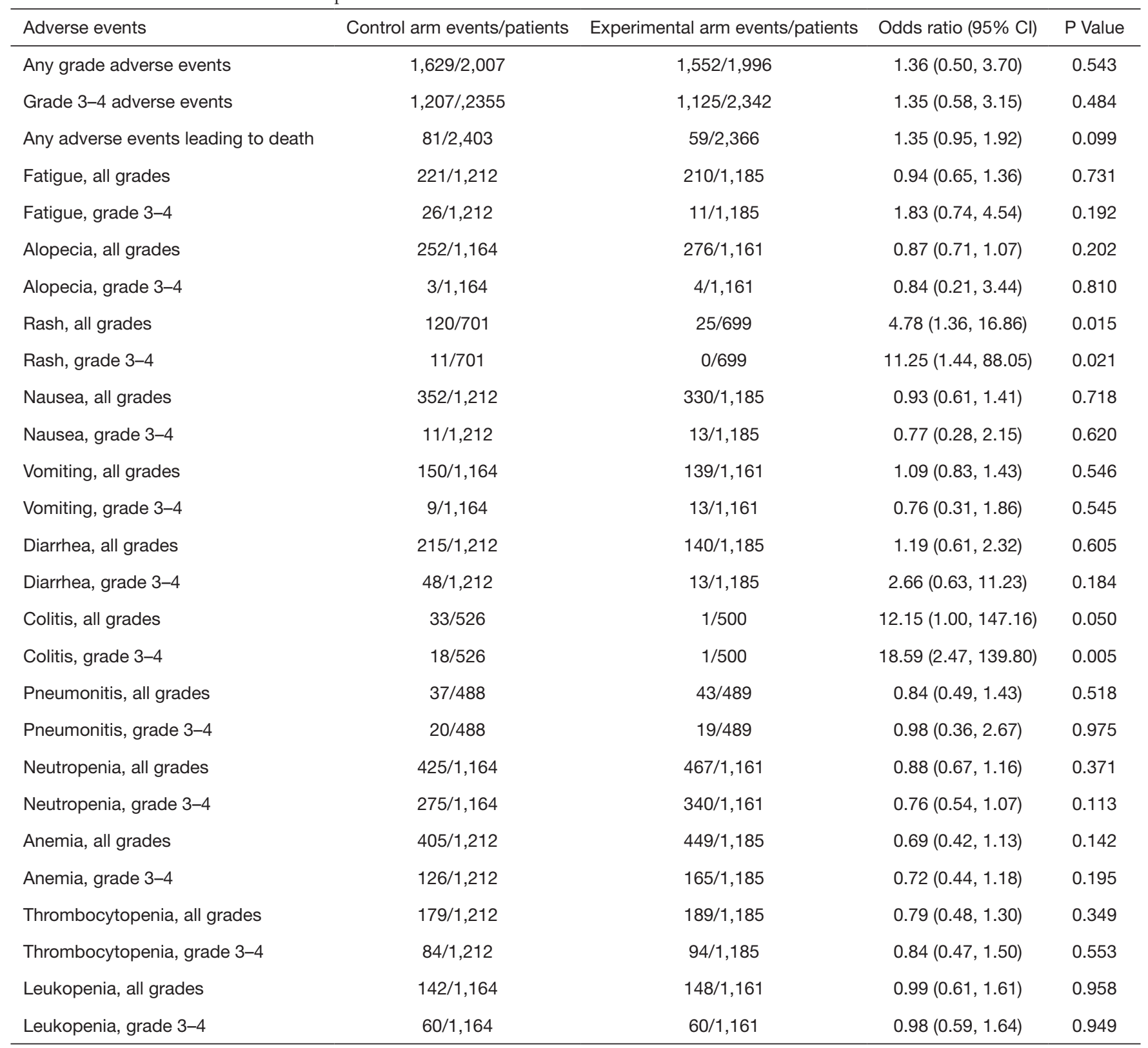

Table 2 shows the incidence of AEs between two groups. As we all know, the ICIs did not increase the frequency AEs for SCLC patients. The overall safety profile was similar between the control and experimental arms. Results showed that for all grades of AEs, the pooled OR was 1.36 (95\% CI: $0.50-3.70 ; \mathrm{P}=0.543)$. As for grades $3-4$ AEs, the $\mathrm{OR}$ was 1.35 (95\% CI: 0.58-3.15; $\mathrm{P}=0.484$ ), and the incidence of fatal due to AEs was 1.35 (95\% CI: 0.95-1.92; $\mathrm{P}=0.099)$. These results were not statistically significant. The most common spectrum of AEs were mainly haematological and gastrointestinal toxicities, and most of toxicities were low grade and controllable. The process was independently conducted by 2 investigators to ensure the accuracy of the results.

\section{Bayesian NMA for indirect comparison of first-line treatment}

Figure 4 shows the network plot established for the NMA 


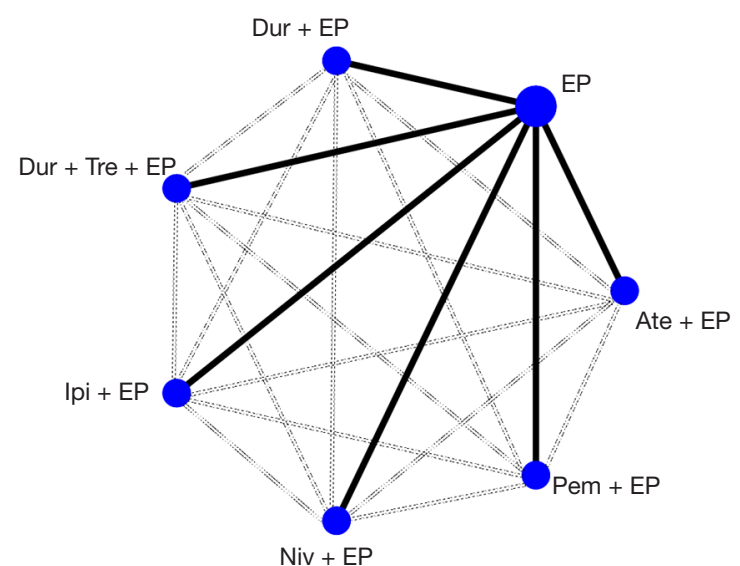

Figure 4 Network structure for first-line therapy of small cell lung cancer. of OS. Each blue circular dot represents each intervention or control group measure. The circle size is proportional to the size of the corresponding sample size of the intervention or the control group. The black lines are proportional to the number of studies for direct comparison. Results of the indirect comparison and the treatment rankings based on SUCRA are presented in Table 3. Overall, the addition of ICIs to standard chemotherapy as first-line therapy in patients with SCLC was associated with a benefit in OS; however, this difference was not statistically significant. The point estimates of the HR for Niv + EP $(0.67,95 \%$ CrI: $0.37,1.20)$, Pem + EP $(0.80,95 \%$ CrI: $0.47,1.40)$, Dur + EP $(0.75,95 \%$ CrI: 0.44, 1.30), Dur + Tre + EP (0.82, 95\% CrI: $0.48,1.40)$, Ate + EP $(0.76,95 \%$ CrI: $0.44,1.30)$, and Ipi + EP $(0.94,95 \%$ CrI: $0.56,1.60)$ were

Table 3 Results of network meta-analysis for overall survival (OS; up) and objective response rate (ORR; down). Each cell contains the pooled hazard ratio or odds ratio with the respective $95 \%$ credible interval

\begin{tabular}{|c|c|c|c|c|c|c|c|}
\hline Experiment & \multicolumn{7}{|c|}{ Comparison } \\
\hline \multirow[t]{2}{*}{$\mathrm{Niv}+\mathrm{EP}$} & - & $0.90(0.40,2.00)$ & $0.88(0.39,2.00)$ & $0.84(0.37,1.90)$ & $0.82(0.37,1.90)$ & $0.71(0.32,1.60)$ & $0.67(0.37,1.20)$ \\
\hline & & $0.79(0.29,2.20)$ & $1.40(0.53,4.00)$ & $0.82(0.30,2.20)$ & $1.20(0.45,3.30)$ & $1.20(0.46,3.30)$ & $1.20(0.56,2.70)$ \\
\hline Dur + EP & $1.10(0.50,2.50)$ & - & $0.99(0.46,2.10)$ & $0.94(0.44,2.00)$ & $0.91(0.54,1.60)$ & $0.80(0.38,1.70)$ & $0.75(0.44,1.30)$ \\
\hline \multirow[t]{2}{*}{ Ate + EP } & $1.10(0.50,2.60)$ & $1.00(0.48,2.20)$ & - & $0.95(0.44,2.00)$ & $0.93(0.44,2.00)$ & $0.81(0.38,1.70)$ & $0.76(0.44,1.30)$ \\
\hline & $0.69(0.25,1.90)$ & $0.55(0.22,1.30)$ & & $0.56(0.23,1.40)$ & $0.83(0.34,2.00)$ & $0.84(0.35,2.00)$ & $0.84(0.44,1.60)$ \\
\hline \multirow[t]{2}{*}{$P e m$ + EP } & $1.20(0.53,2.70)$ & $1.10(0.50,2.30)$ & $1.10(0.49,2.30)$ & - & $0.98(0.46,2.10)$ & $0.85(0.40,1.80)$ & $0.80(0.47,1.40)$ \\
\hline & $1.20(0.45,3.40)$ & $0.97(0.40,2.40)$ & $1.80(0.71,4.40)$ & & $1.50(0.61,3.60)$ & $1.50(0.63,3.60)$ & $1.50(0.78,2.80)$ \\
\hline \multirow[t]{2}{*}{$I p i+E P$} & $1.40(0.63,3.10)$ & $1.30(0.60,2.60)$ & $1.20(0.59,2.70)$ & $1.20(0.56,2.50)$ & $1.1(0.55,2.40)$ & - & $0.94(0.56,1.60)$ \\
\hline & $0.82(0.31,2.20)$ & $0.65(0.28,1.50)$ & $1.20(0.50,2.80)$ & $0.67(0.28,1.60)$ & $0.98(0.42,2.30)$ & & $0.99(0.56,1.80)$ \\
\hline \multirow[t]{2}{*}{ EP } & $1.50(0.81,2.70)$ & $1.30(0.78,2.30)$ & $1.30(0.77,2.30)$ & $1.20(0.73,2.10)$ & $1.20(0.72,2.10)$ & $1.1(0.63,1.80)$ & - \\
\hline & $0.83(0.37,1.80)$ & $0.65(0.35,1.20)$ & $1.20(0.63,2.30)$ & $0.67(0.35,1.30)$ & $0.99(0.53,1.80)$ & $1.0(0.56,1.80)$ & \\
\hline \multirow[t]{2}{*}{ SUCRA value } & 0.7393 & 0.6390 & 0.6131 & 0.5382 & 0.4956 & 0.3069 & 0.1681 \\
\hline & 0.5782 & 0.8100 & 0.3660 & 0.7700 & 0.3821 & 0.3810 & 0.2127 \\
\hline
\end{tabular}

Note: SUCRA, the surfaces under the cumulative ranking curve. EP, etoposide and platinum-based drugs. Pem + EP, pembrolizumab combined with EP. Ate + EP, atezolizumab combined with EP. Ipi + EP, ipilimumab combined with EP. Dur + Tre + EP, durvalumab and tremelimab combined with EP. Dur + EP, durvalumab combined with EP, and Niv + EP, nivolumab combined with EP. 
not significant compared with EP alone. Ranking of all interventions included in the NMA for OS indicated that Niv + EP (SUCRA 0.7393) was most likely to be the best treatment, while Dur + EP (SUCRA 0.6390) and Ate + EP (SUCRA 0.6131) were ranked the second-best and thirdbest regimens, respectively.

Similar results were found in ORR. Compared with EP, the OR for Niv + EP (1.20, 95\% CrI: 0.56, 2.70), Pem + EP (1.50, 95\% CrI: 0.78, 2.80), Dur + EP (1.50, 95\% CrI: $0.82,2.80)$, Dur + Tre + EP (1.00, 95\% CrI: 0.54, 1.90), Ate + EP (0.84, 95\% CrI: 0.44, 1.60), and Ipi + EP (0.99, 95\% CrI: $0.56,1.80)$ were not significant. Dur + EP appeared to be inferior to all competing interventions. Ranks for interventions indicated that Dur + EP had a rank of being the best treatment regimen, and was associated with an $81 \%$ probability; this was followed by Pem + EP (77\%), and Niv $+\mathrm{EP}(57.82 \%)$ respectively.

\section{Quality assessment, publication bias and sensitivity analysis}

The result of quality assessment showed all studies were low risk of bias in random sequence generation, allocation concealment and blinding of outcome assessment and 3 studies were high risk of bias in blinding of participants and personnel (Figure S1). To detect publication bias, we carried out funnel plot and Egger's test and demonstrated no obvious publication bias existed (Figure S2). To evaluate the robustness of the outcomes, eight treatments were included for sensitivity analysis. The results were stable after omitting each study (Figure S3).

\section{Inconsistency assessment}

Deviance information criteria (DIC) is widely used to assess the goodness of fit of the models in Bayesian frameworks. In our study, DIC were calculated in an inconsistent model (DIC $=12.02)$ and in a consistent model (DIC $=11.99)$. Our result suggested that the data were considered to basically conform to the consistency and there is little to choose between the two models because the difference was less than 5 (37).

\section{Discussion}

The biological invasiveness of the cancer and the lack of effective treatment options are the main reasons for the dismal survival of SCLC patients. Compared to the recent improvements in non-small cell lung cancer (NSCLC) therapies, the management for SCLC has not been significantly improved since it began about 30 years ago. Platinum doublet has been the standard therapy for patients with SCLC. However, ICIs have emerged in recent RCTs as a new option for these patients. Through direct comparison, some meaningful results can be inferred for SCLC, but the benefits are slight. In the trials of ICIs combined with EP as first-line treatment of ES-SCLC, the results showed that added ICIs were beneficial for OS and PFS. On the contrary, there were no benefit observed with ICIs drugs as maintenance or second-line therapy. Because no direct comparison yet exists between different ICI drugs, we conducted an NMA and found that Niv + EP may generally be preferred for OS in first-line treatments, but for ORR, Dur + EP therapy was found to be superior. However, these data were not statistically significant. In short, there is no clear evidence for a significant difference in efficacy between ICI drugs for first-line therapy.

As for the role of neoadjuvant chemotherapy, briefly, the NCCN (National Comprehensive Cancer Network) Guidelines state that patients with limited-stage IIB to IIIC (T3-4, N0, M0; T1-4, N1-3, M0) do not benefit from surgery (38). However, recent data from numerous retrospective studies have demonstrated neoadjuvant chemotherapy combined with surgery may could give the LS-SCLC patients a better chance of survival. In Hara's case series (39), stage III survival tended to be better in patients with preoperative chemotherapy (median survival time, 29 months) than in those who had had postoperative chemotherapy only (median survival time, 17 months). For resectable stage III, particularly in patients with N2 disease, adjuvant resection after chemotherapy may be a favorable choice in the management of SCLC. Xu et al. (40) also reported neoadjuvant chemotherapy combined with surgery provided reasonable options for pIIIa-N2 LS-SCLC patients, which can give them a better chance of survival. But the controversy about neoadjuvant chemotherapy for LS-SCLC still exists. There also have studies which showed that compared with adjuvant chemotherapy combined with surgery, neoadjuvant chemotherapy combined with surgery has no better survival (41). Therefore, the efficacy of the comprehensive treatment including neoadjuvant chemotherapy and/or radiotherapy for LS-SCLC patients needs to be confirmed with a larger sample size and multicenter research results. A prospective study conducted by Veronesi et al. (42) supported the conclusion that patients' survival is related to tumor response to neoadjuvant 
chemotherapy and that complete responders had a significantly longer survival than partial responders and nonresponders. Lucchi et al. (43) also showed there was a significant correlation between survival and lymph node status. Thus far, there are no data to support the use of immunotherapy before surgery in patients with LS-SCLC.

About 2/3 SCLC patients have extensive stage at the time of diagnosis, with a high degree of malignancy and early occurrence of lymphatic and hematogenous metastasis. Previous studies have indicated that patients with the extensive disease have a poor prognosis with a 2-year survival close to $5 \%$ and median survival of less than 1 year. Younger age, good PS, female patients and a single metastatic site are favorable prognostic factors in patients with extensive-stage disease. But these studies still had some limitations and further studies involving large sample with complete clinical data are warranted $(44,45)$.

We conducted the subgroup analysis of the baseline characteristics for the population. For age subgroup, we used 65 years as the cut-off age because a number of large clinical trials showed that patients older than 65 years responded more efficiently to ICIs compared with younger patients (46), but some studies found no differences between age groups (47). Our meta-analysis showed that ICIs combined with chemotherapy can significantly improve OS in both younger and older patients compared with controls. The results of subgroup analysis also showed that patients with brain metastases and those who do not smoke, may not benefit from immunotherapy. Brain metastasis frequently has a threat to the quality of life for SCLC patients and has a poor prognosis than those without brain metastases. And due to the existence of the blood-brain barrier, it is difficult for chemotherapy agents to cross from the circulatory system to the central nervous system (48). The benefits of immunotherapy on brain metastasis is still unclear. Although lymphocytes that exist outside the brain are activated, and the activated lymphocytes can pass through the blood-brain barrier to the brain, the effect is limited because the tumor microenvironment of the brain metastases is immunosuppressed and complicated (49). Our study showed the effect of immunotherapy on brain metastasis may also have a limited effect on brain metastases. As previously mentioned, there is an association between tumor mutation burden and benefit to receiving immunotherapy (50). Cigarette smoke may initiate high tumor mutation burden in patients, so it is easier to benefit from immunotherapy for smokers than those who do not smoke (5).
The immunotherapy for SCLC is still developing, as evidenced by the limited benefit and a lack of predictive biomarkers. The key factor in the future development of ICIs will be the identification of biomarkers which can predict if patients are suitable for ICI therapy. The Keynote-604 study showed the PFS and OS HRs were similar in participants with PD-L1-positive and PD-L1negative tumors. And the Impower-133 study showed no clear suggestion that blood-based tumor mutational burden levels at either cutoff (10 or 16 mutations per megabase) were predictive of benefit with ICIs. Because of the lack of adequate data, we cannot produce subgroup analysis according to the level of PD-L1 expression and tumor mutational burden (TMB). Therefore, the level of PDL1 expression and TMB in SCLC have failed to achieve this goal, and the use of PD-L1 or TMB as predictive biomarkers for immunotherapy is not recommended (51). Generally speaking, the effect of ICI single-agent therapy is limited, and the combination therapy may be the best choice. There are increasing studies to explore the ICIs combined with cytotoxic drugs, anti-angiogenic drugs or radiotherapy, which could have advantages for patients survival in some malignancies. However, the time and sequence of the combination therapy still need to be further studied. And the incidence of AEs caused by multiple therapies will increase, which requires our clinicians to pay closely attention.

Immunotherapy also has certain adverse reactions, namely immune-related adverse events (irAEs), which are mainly caused by the activation of the body's normal anti-tumor immune response by immunotherapy. IrAEs are toxicities associated with checkpoint inhibitors that are autoimmune or autoinflammatory in origin. Immune therapy have longterm effects on other normal tissues, so patients may present with irAEs late in the course of treatment, and-in some cases-months or even years after treatment discontinuation. For example, type 1 diabetes and inflammatory arthritis persist beyond the cessation of ICIs (52). Gaining a better understanding of irAEs is also critical for the advancement of immunotherapy, as this type of toxicity is largely unknown to oncologists. Although serious irAEs are still rare (about $10 \%$ under ICI monotherapy), they may be life-threatening if they are not detected and managed properly (53). Patients should be closely monitored throughout immunotherapy even in the follow-up course. Physical examination (such as dermatologic examination), Laboratory examination (such as blood tests), imaging examination (pulmonary tests) and Cardiac tests performed 
will be necessary. Since some of the toxicities appeared late, even after the ICIs treatment discontinuation, it is also very important to follow up the above-mentioned examinations and test items after treatment. Especially the indicators of kidney function, thyroid function, pituitary function and so on. It is currently considered that patients should be monitored for symptoms for at least 1 year after the end of ICIs treatment (54).

Other therapy strategies are being recently explored, including rovalpituzumab tesirine (Rova-T) was designed to specifically target delta-like ligand 3 (DLL3) and promote DNA damage and tumor cell death in small cell lung cancer. A phase II clinical trial showed that Rova- $T$ was not active in relapsed and refractory small cell lung cancer (55). Veliparib, a poly (ADP ribose) polymerase inhibitor, potentiated standard chemotherapy against SCLC. The results of ECOG-ACRIN 2511 study showed that the addition of veliparib on the basis of first-line chemotherapy for ES-SCLC had a certain efficacy, and the study met its prespecified end point (56). Lurbinectedin is a selective inhibitor of oncogenic transcription and inhibits oncogenic transcription, leading to tumour cell apoptosis. The study showed Lurbinectedin was active as second-line therapy for SCLC after failure of platinum-based chemotherapy and this treatment was effective and safe (57). In addition, chimeric antigen receptor (CAR) $\mathrm{T}$ cells, tumor vaccines, antibody-drug conjugates (ADCs) and immunomodulators also have some benefits in a way. With the increasing study of proteomics and transcriptomics of various genetic signaling pathways and exploration of targeted therapy and immunotherapy, there are more and more options for small cell lung cancer, and the benifits of survival is gradually improved.

In clinical practice, we are often faced with complicated situations. Patients may be weak, or have multiple complications, or concern about the cost of treatment. These factors will affect our final treatment options and the effect of therapy. But our study ignored these issues such as the availability and cost of drugs. Therefore, we cannot completely follow the results of this study to care patients in clinical practice. We still need to comprehensive consider the best treatment based on the actual situation of the patients.

Our meta-analysis involved a few limitations. First, the interventions examined in this study do not include the unpublished data of SCLC treatment studies. This may be a rapidly growing source of data, as the number of formally approved interventions is increasing. For example, the
CheckMate-451 and ECOG-ACRIN EA5161 trials are ongoing, and researchers have only reported immature OS and PFS data thus far. Second, there was some variability between the trials included in this study, such as baseline characteristics for patients, chemotherapy regimens etc., which might have produced heterogeneity and inaccuracy. Finally, this is a comprehensive analysis of published results, not individual patient data. These limitations prevent us from drawing clear conclusions that can be used as a evidence for clinical practice.

\section{Conclusions}

This meta-analysis summarized all of the current evidence, and supports ICIs as valuable component of SCLC treatment. Our results showed that ICIs combined with EP as first-line treatment of SCLC were more beneficial for OS and PFS than standard chemotherapy alone. On the contrary, there were no benefits observed with ICIs drugs used as maintenance or second-line therapy, and no obvious evidence showing significant differences in the efficacy between the different ICI drugs used for the first-line therapy. As for toxicity, the ICIs did not increase the frequency AEs for SCLC patients. However, as some studies are ongoing and the full data have still not been reported, our conclusions may not be completely representative.

\section{Acknowledgments}

The authors appreciate the academic support from the AME Lung Cancer Collaborative Group.

Funding: Mechanism of bone metastasis enhanced by M2 polarization via TGF- $\beta 1$-induced NGF/TrkA pathway upregulation in prostate cancer, Project supported by the National Natural Science Foundation of China (no. 81872101).

\section{Footnote}

Reporting Checklist: The authors have completed the PRISMA reporting checklist. Available at http://dx.doi. org/10.21037/atm-21-1423

Conflicts of Interest: All authors have completed the ICMJE uniform disclosure form (available at http://dx.doi. org/10.21037/atm-21-1423). The authors have no conflicts of interest to declare. 
Ethical Statement: The authors are accountable for all aspects of the work in ensuring that questions related to the accuracy or integrity of any part of the work are appropriately investigated and resolved.

Open Access Statement: This is an Open Access article distributed in accordance with the Creative Commons Attribution-NonCommercial-NoDerivs 4.0 International License (CC BY-NC-ND 4.0), which permits the noncommercial replication and distribution of the article with the strict proviso that no changes or edits are made and the original work is properly cited (including links to both the formal publication through the relevant DOI and the license). See: https://creativecommons.org/licenses/by-nc-nd/4.0/.

\section{References}

1. Jett JR, Schild SE, Kesler KA, et al. Treatment of small cell lung cancer: Diagnosis and management of lung cancer, 3rd ed: American College of Chest Physicians evidencebased clinical practice guidelines. Chest 2013;143:e400Se419S.

2. Micke P, Faldum A, Metz T, et al. Staging small cell lung cancer: Veterans Administration Lung Study Group versus International Association for the Study of Lung Cancer What limits limited disease? Lung Cancer 2002;37:271-6.

3. Luo J, Xu L, Zhao L, et al. Timing of thoracic radiotherapy in the treatment of extensive-stage small-cell lung cancer: important or not. Radiat Oncol 2017;12:42.

4. Kalemkerian GP. Advances in the treatment of small-cell lung cancer. Semin Respir Crit Care Med 2011;32:94-101.

5. Alexandrov LB, Nik-Zainal S, Wedge DC, et al. Signatures of mutational processes in human cancer. Nature 2013;500:415-21.

6. Reck M, Heigener D, Reinmuth N. Immunotherapy for small-cell lung cancer: emerging evidence. Future Oncol 2016;12:931-43.

7. Koyama K, Kagamu H, Miura S, et al. Reciprocal CD4+ T-cell balance of effector CD62Llow CD4+ and CD62LhighCD25+ CD4+ regulatory $\mathrm{T}$ cells in small cell lung cancer reflects disease stage. Clin cancer Res 2008;14:6770-9.

8. Ricciuti B, Kravets S, Dahlberg SE, et al. Use of targeted next generation sequencing to characterize tumor mutational burden and efficacy of immune checkpoint inhibition in small cell lung cancer. J Immunother cancer 2019;7:87.

9. Pardoll DM. The blockade of immune checkpoints in cancer immunotherapy. Nat Rev Cancer 2012;12:252-64.

10. Ishii H, Azuma K, Kawahara A, et al. Significance of programmed cell death-ligand 1 expression and its association with survival in patients with small cell lung cancer. J Thorac Oncol 2015;10:426-30.

11. Ready NE, Ott PA, Hellmann MD, et al. Nivolumab Monotherapy and Nivolumab Plus Ipilimumab in Recurrent Small Cell Lung Cancer: Results From the CheckMate 032 Randomized Cohort. J Thorac Oncol 2020;15:426-35.

12. Antonia SJ, López-Martin JA, Bendell J, et al. Nivolumab alone and nivolumab plus ipilimumab in recurrent smallcell lung cancer (CheckMate 032): a multicentre, openlabel, phase 1/2 trial. Lancet Oncol 2016;17:883-95.

13. Reck M, Vicente D, Ciuleanu T, et al. LBA5 - Efficacy and safety of nivolumab (nivo) monotherapy versus chemotherapy (chemo) in recurrent small cell lung cancer (SCLC): Results from CheckMate 331. Ann Oncol 2018;29:x43.

14. Owonikoko TK, Kim HR, Govindan R, et al. Nivolumab (nivo) plus ipilimumab (ipi), nivo, or placebo (pbo) as maintenance therapy in patients (pts) with extensive disease small cell lung cancer (ED-SCLC) after first-line (1L) platinum-based chemotherapy (chemo): Results from the double-blind, rando. Ann Oncol 2019;30:ii77.

15. Leal T, Wang Y, Dowlati A, et al. Randomized phase II clinical trial of cisplatin/carboplatin and etoposide (CE) alone or in combination with nivolumab as frontline therapy for extensive-stage small cell lung cancer (ES-SCLC): ECOG-ACRIN EA5161. J Clin Oncol 2020;38:abstr 9000.

16. Horn L, Mansfield AS, Szczęsna A, et al. First-line atezolizumab plus chemotherapy in extensive-stage smallcell lung cancer. N Engl J Med 2018;379:2220-9.

17. Reck M, Liu SV, Mansfield AS, et al. IMpower133: Updated overall survival (OS) analysis of first-line (1L) atezolizumab (atezo) + carboplatin + etoposide in extensivestage SCLC (ES-SCLC). Ann Oncol 2019;30:v710-1.

18. Rudin CM, Awad MM, Navarro A, et al. Pembrolizumab or Placebo Plus Etoposide and Platinum as First-Line Therapy for Extensive-Stage Small-Cell Lung Cancer: Randomized, Double-Blind, Phase III KEYNOTE-604 Study. J Clin Oncol 2020;38:2369-79.

19. Rudin CM, Awad MM, Navarro A, et al. KEYNOTE-604: Pembrolizumab (pembro) or placebo plus etoposide and platinum (EP) as first-line therapy for extensive-stage (ES) small-cell lung cancer (SCLC). J Clin Oncol 2020;38:abstr 9001. 
20. Pujol JL, Greillier L, Audigier-Valette C, et al. A Randomized Non-Comparative Phase II Study of AntiProgrammed Cell Death-Ligand 1 Atezolizumab or Chemotherapy as Second-Line Therapy in Patients With Small Cell Lung Cancer: Results From the IFCT-1603 Trial. J Thorac Oncol 2019;14:903-13.

21. Nishio M, Sugawara S, Atagi S, et al. Subgroup Analysis of Japanese Patients in a Phase III Study of Atezolizumab in Extensive-stage Small-cell Lung Cancer (IMpower133). Clin Lung Cancer 2019;20:469-76.e1.

22. Paz-Ares LG, Dvorkin M, Chen Y, et al. Durvalumab \pm tremelimumab + platinum-etoposide in first-line extensivestage SCLC (ES-SCLC): Updated results from the phase III CASPIAN study. J Clin Oncol 2020;38:abstr 9002.

23. Paz-Ares L, Dvorkin M, Chen Y, et al. Durvalumab plus platinum-etoposide versus platinum-etoposide in firstline treatment of extensive-stage small-cell lung cancer (CASPIAN): a randomised, controlled, open-label, phase 3 trial. Lancet 2019;394:1929-39.

24. Reck M, Luft A, Szczesna A, et al. Phase III randomized trial of ipilimumab plus etoposide and platinum versus placebo plus etoposide and platinum in extensive-stage small-cell lung cancer. J Clin Oncol 2016;34:3740-48.

25. Hellmann MD, Callahan MK, Awad MM, et al. Tumor Mutational Burden and Efficacy of Nivolumab Monotherapy and in Combination with Ipilimumab in Small-Cell Lung Cancer. Cancer Cell 2018;33:853-61.e4.

26. Goldman JW, Dvorkin M, Chen Y, et al. Articles Durvalumab, with or without tremelimumab, plus platinum - etoposide versus platinum - etoposide alone in first-line treatment of extensive-stage small-cell lung cancer (CASPIAN): updated results from a randomised, controlled, open-label, phase 3 trial. Lancet Oncol 2021;22:51-65.

27. Niu ZC, Wang ZY, Li X. Comparative effectiveness of immune checkpoint inhibitors for treatment of small-cell lung cancer: a network meta-analysis. PROSPERO 2020 CRD42020211223. Available online: https://www.crd.york.ac.uk/prospero/display_record. php?ID=CRD42020211223

28. Liberati A, Altman DG, Tetzlaff J, et al. The PRISMA Statement for Reporting Systematic Reviews and Meta-Analyses of Studies That Evaluate Health Care Interventions: Explanation and Elaboration. J Clin Epidemiol 2009;62:e1-34.

29. Higgins JPT, Altman DG, Gøtzsche PC, et al. The Cochrane Collaboration's tool for assessing risk of bias in randomised trials. BMJ 2011;343:d5928.
30. DerSimonian R, Laird N. Meta-analysis in clinical trials. Control Clin Trials 1986;7:177-88.

31. Higgins JPT, Thompson SG, Deeks JJ, et al. Measuring inconsistency in meta-analyses. BMJ 2003;327:557-60.

32. Caldwell DM, Ades AE, Higgins JPT. Simultaneous comparison of multiple treatments: combining direct and indirect evidence. BMJ 2005;331:897-900.

33. Dias S, Sutton AJ, Ades AE, et al. Evidence Synthesis for Decision Making 2 : A Generalized Linear Modeling Framework for Pairwise and Network Meta-analysis of Randomized Controlled Trials. Med Decis Making 2013;33:607-17.

34. Gelman BA. General Methods for Monitoring Convergence of Iterative Simulations. J Comput Graph Stat 1998;7:434-55.

35. Cope S, Jansen JP. Quantitative summaries of treatment effect estimates obtained with network meta-analysis of survival curves to inform decision-making. BMC Med Res Methodol 2013;13:147.

36. Neupane B, Richer D, Bonner AJ, et al. Network metaanalysis using R: a review of currently available automated packages. PLoS One 2014;9:e115065.

37. Dias S, Welton NJ, Caldwell DM, et al. Checking consistency in mixed treatment comparison meta-analysis. Stat Med 2010;29:932-44.

38. Lad T, Piantadosi S, Thomas P, et al. A prospective randomized trial to determine the benefit of surgical resection of residual disease following response of small cell lung cancer to combination chemotherapy. Chest 1994;106:320S-3S.

39. Hara N, Ohta M, Ichinose $Y$, et al. Influence of surgical resection before and after chemotherapy on survival in small cell lung cancer. J Surg Oncol 1991;47:53-61.

40. Xu YJ, Zheng $\mathrm{H}$, Gao W, et al. Is neoadjuvant chemotherapy mandatory for limited-disease smallcell lung cancer? Interact Cardiovasc Thorac Surg 2014;19:887-93.

41. Dou X, Wang Z, Wang L, et al. Analysis of efficacy of surgical treatment for a small cell lung cancer. Zhongguo Fei Ai Za Zhi 2017;20:88-92.

42. Veronesi G, Scanagatta P, Leo F, et al. Adjuvant surgery after carboplatin and VP16 in resectable small cell lung cancer. J Thorac Oncol 2007; 2:131-4.

43. Lucchi M, Mussi A, Chella A, et al. Surgery in the management of small cell lung cancer. Eur J Cardiothorac Surg 1997;12:689-93.

44. Wheatley-Price P, Ma C, Ashcroft LF, et al. The strength of female sex as a prognostic factor in small-cell lung 
cancer: a pooled analysis of chemotherapy trials from the Manchester Lung Group and Medical Research Council Clinical Trials Unit. Ann Oncol 2010;21:232-7.

45. Cai Q, Luo HL, Gao XC, et al. Clinical features and prognostic factors of small cell lung cancer: A retrospective study in 148 patients. J Huazhong Univ Sci Technolog Med Sci 2016;36:916-22.

46. Wu Q, Wang Q, Tang X, et al. Correlation between patients' age and cancer immunotherapy efficacy. Oncoimmunology 2019;8:e1568810.

47. Yang F, Markovic SN, Molina JR, et al. Association of Sex, Age, and Eastern Cooperative Oncology Group Performance Status With Survival Benefit of Cancer Immunotherapy in Randomized Clinical Trials A Systematic Review and Meta-analysis. JAMA Netw Open 2020;3:e2012534.

48. Castrucci WA, Knisely JPS. An Update on the Treatment of CNS Metastases in Small Cell Lung Cancer. Cancer J 2008;14:138-46.

49. Quail DF, Joyc JA. The Microenvironmental Landscape of Brain Tumors. Cancer Cell 2017;31:326-41.

50. Hellmann MD, Nathanson T, Rizvi H, et al. Genomic Features of Response to Combination Immunotherapy in Patients with Advanced Non-Small-Cell Lung Cancer. Cancer Cell 2018;33:843-52.e4.

51. Hellmann MD, Callahan MK, Awad MM, et al. Tumor Mutational Burden and Efficacy of Nivolumab Monotherapy and in Combination with Ipilimumab in Small-Cell Lung Cancer. Cancer Cell 2019;35:329.

Cite this article as: Niu Z, Guo S, Cao J, Zhang Y, Guo X, Grossi F, Ichiki Y, Li Y, Wang Z. Immune checkpoint inhibitors for treatment of small-cell lung cancer: a systematic review and meta-analysis. Ann Transl Med 2021;9(8):705. doi: 10.21037/ atm-21-1423
52. Calabrese LH, Calabrese C, Cappelli LC. Rheumatic immune-related adverse events from cancer immunotherapy. Nat Rev Rheumatol 2018;14:569-79.

53. Khan Z, Hammer C, Guardino E, et al. Mechanisms of immune-related adverse events associated with immune checkpoint blockade: using germline genetics to develop a personalized approach. Genome Med 2019;11:39.

54. Puzanov I, Diab A, Abdallah K, et al. Managing toxicities associated with immune checkpoint inhibitors: consensus recommendations from the Society for Immunotherapy of Cancer (SITC) Toxicity Management Working Group. J Immunother Cancer 2017;5:95.

55. Morgensztern D, Besse B, Greillier L, et al. Efficacy and safety of rovalpituzumab tesirine in third-line and beyond patients with DLL3-expressing, relapsed/refractory smallcell lung cancer: results from the phase II TRINITY study. Clin Cancer Res 2019;25:6958-66.

56. Owonikoko TK, Dahlberg SE, Sica GL, et al. Randomized phase II trial of cisplatin and etoposide in combination with veliparib or placebo for extensive-stage small-cell lung cancer: ECOG-ACRIN 2511 study. J Clin Oncol 2019;37:222-9.

57. Trigo J, Subbiah V, Besse B, et al. Lurbinectedin as secondline treatment for patients with small-cell lung cancer: a single-arm, open-label, phase 2 basket trial. Lancet Oncol 2020;21:645-54.

(English Language Editor: J. Gray) 


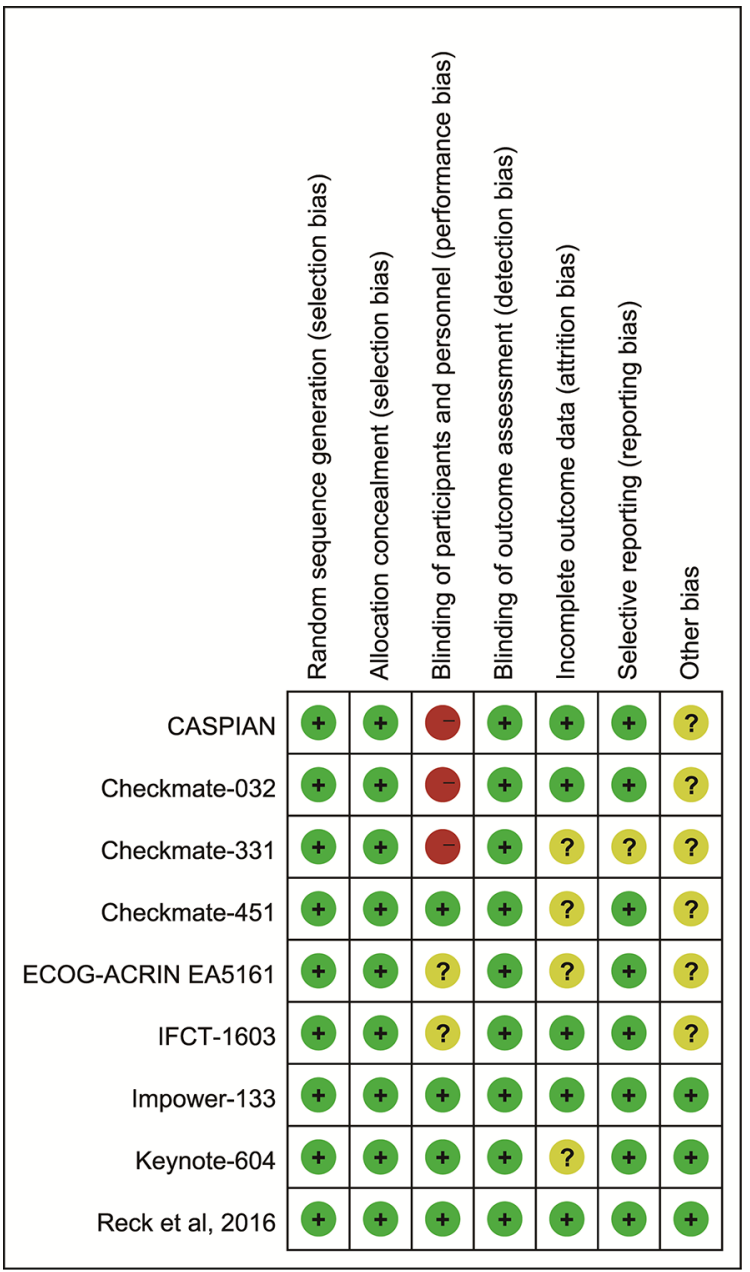

Figure S1 Risk of bias summary. Review of authors' judgements about each risk of bias item for each included study. "+" low risk of bias; "?" unevaluable risk of bias; "_" high risk of bias.

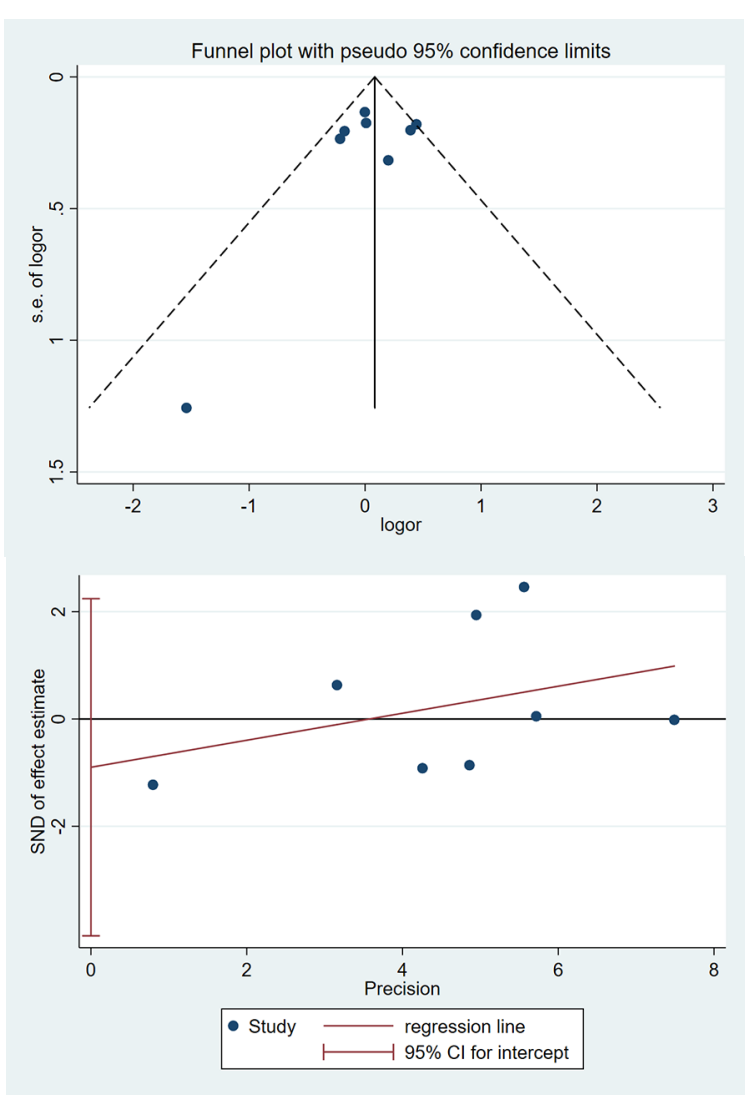

Figure S2 Funnel plot and Egger's test for publication bias.

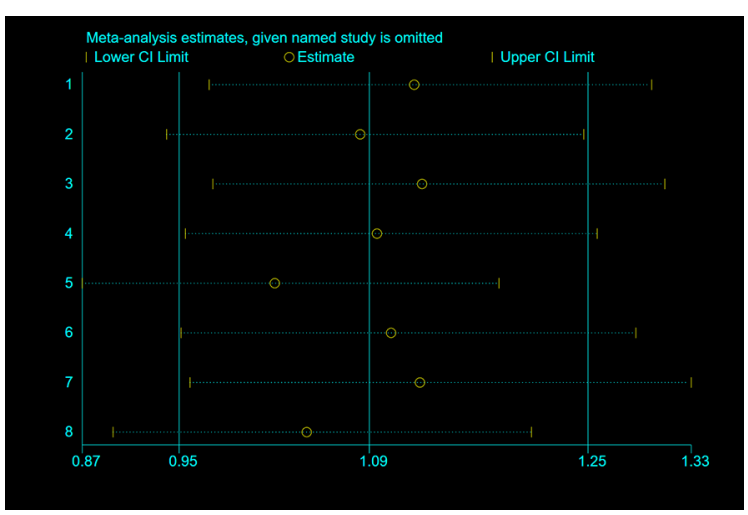

Figure S3 Sensitivity analysis. 
Table S1 Search strategies

Search strategies in PubMed

\#1 Small Cell Lung Carcinoma[mh]

\#2 Small Cell Lung Cancer [tiab] OR Oat Cell Lung Cancer [tiab] OR Small Cell Cancer of the Lung [tiab] OR Carcinoma, Small Cell Lung [tiab] OR Oat Cell Carcinoma of Lung [tiab]

\#3 \#1 OR \#2

\#4 Immune Checkpoint Inhibitors [mh] OR Immune Checkpoint Blockers [tiab] OR PD-L1 Inhibitors [tiab] OR Programmed Death-Ligand 1 Inhibitors [tiab] OR CTLA-4 Inhibitors [tiab] OR Cytotoxic T-Lymphocyte-Associated Protein 4 Inhibitors [tiab] OR PD-1 Inhibitors [tiab] OR Programmed Cell Death Protein 1 Inhibitor [tiab] OR Nivolumab [tiab] OR Atezolizumab [tiab] OR Durvalumab [tiab] OR Camrelizumab [tiab] OR Sintilimab [tiab] OR Avelumab [tiab] OR Ipilimumab [tiab] OR Tremelimumab [tiab]

\#5 Randomized Controlled Trial [pt] OR Controlled Clinical Trial [pt] OR Randomized [tiab] OR Placebo [tiab] OR Clinical Trials as topic [mesh: noexp] or Randomly [tiab] OR Trial [ti] NOT animals [mh] NOT humans [mh]

\#6 \#3 AND \#4 AND \#5

Search strategies in Cochrane Library

\#1 MeSH descriptor:[Small Cell Lung Carcinoma] explode all trees

\#2 (Small Cell Lung Carcinoma OR Small Cell Lung Cancer OR Oat Cell Lung Cancer OR Small Cell Cancer of the Lung OR Carcinoma, Small Cell Lung OR Oat Cell Carcinoma of Lung):ti,ab,kw

\#3 \#1 OR \#2

\#4 (Immune Checkpoint Inhibitors OR Immune Checkpoint Blockers OR PD-L1 Inhibitors OR Programmed Death-Ligand 1 Inhibitors OR CTLA-4 Inhibitors OR Cytotoxic T-Lymphocyte-Associated Protein 4 Inhibitors OR PD-1 Inhibitors OR Programmed Cell Death Protein 1 Inhibitor OR Nivolumab OR Atezolizumab OR Durvalumab OR Camrelizumab OR Sintilimab OR Avelumab OR Ipilimumab OR Tremelimumab):ti,ab,kw

\#5 \#3 AND \#4 